\title{
Film and Pore Diffusion Modeling for Adsorption of Reactive Red 2 from Aqueous Solution on to Activated Carbon Prepared from Bio-Diesel Industrial Waste
}

\author{
S. KARTHIKEYAN*, B. SIVAKUMAR ${ }^{\S}$ and N.SIVAKUMAR \\ Department of Chemistry \\ Chikkanna Government Arts College, Tirupur, Tamilnadu, India \\ ${ }^{\S}$ Department of Chemistry \\ Angel College of Engineering and Technology, Tirupur, Tamilnadu, India \\ skmush@rediffmail.com
}

Received 12 March 2010; Accepted 23 May 2010

\begin{abstract}
The bio-diesel industrial waste, Pongamia pinnata seed shell was utilized as a potential adsorbent for the toxic textile dye 'Reactive Red 2'. Preliminary information was gathered by batch mode contact time adsorption studies, which include effect of $\mathrm{pH}$, contact time, dye concentration and temperature. Plausible mechanism of the on going adsorption process and thermodynamic parameters involved were obtained by carrying out kinetic measurements. To identify whether the on going process is particle diffusion or film diffusion, the treatment given by Boyd and Reichenberg was employed.
\end{abstract}

Keywords: Bio-diesel industrial waste, Pongamia pinnata seed shell, Reactive Red 2, Particle diffusion, Film diffusion.

\section{Introduction}

Commercially available activated carbons are expensive and their use requires elaborate regeneration and reactivation procedure. The gradual loss of activated carbon during regeneration can be materially affecting the economic viability of the process. The use of structured and robust activated carbons based on expensive starting material is unjustified. Therefore, other sources for materials far, activated carbons are explored. Cellulose and lignocellulosic wastes have long been recognized as starting materials for the preparation activated carbon ${ }^{1}$.

This has led many workers to search for cheaper sources to prepare activated carbons or cheaper substitutes like fly-ash ${ }^{2}$, wollastonite ${ }^{3}$, sawdust, rice husk and coir pith carbon ${ }^{4}$, turmeric waste, Jatropha curcus seed shell, Delomix regia seed shell and Ipomea carnia stem are the materials which have been already successfully studied for removal of color and 
organic matters ${ }^{5}$. Pongamia pinnata is one among the few nitrogen fixing trees to produce seeds containing $30-40 \%$ oil. It is often planted as an ornamental tree but now-a-days it is considered as alternative source for bio-diesel.

Textile effluents are known toxicants, which inflict acute disorders in aquatic organisms uptake of textile effluents through food chain in aquatic organisms may cause various physiological disorders like hypertension, sporadic fever, renal damage, cramps etc. ${ }^{6}$, Reactive Red 2 is one such effluent which causes various disorders when present in aqueous solution. Hence the treatment of wastewater containing dye is a challenging problem ${ }^{7}$.

In the present investigation the adsorption of Reactive Red 2 on activated carbon prepared from pongamia pinnata pod shell waste by carbonization with sulphuric acid has been achieved. The kinetic and equilibrium adsorption data obtained were utilized to characterize the sample prepared ${ }^{8}$.

The amounts and rates of adsorption of Reactive Red 2 using above activated carbon from water were then measured. Three simplified kinetic models including pseudo first order, Pseudo second order equations and Elovich equations were used to describe the adsorption process.

\section{Experimental}

Pongamia pinnata seed shell waste was collected from local area of Erode district, Tamilnadu, India. It was dried, charred with excess quantity of concentrated sulphuric acid keeping at $120{ }^{\circ} \mathrm{C}$ for 10 hours. Then the resultant carbon was washed with excess quantity of distilled water and dried at $110{ }^{\circ} \mathrm{C}$ for 1 hour and the material obtained was soaked in $5 \%$ sodium bicarbonate solution and allowed to stand overnight to remove any residual acid. The material was thoroughly washed with hot distilled water until washings were nearly of neutral effect. To eliminate surface groups and thermal activation, the carbonized material was treated at $800{ }^{\circ} \mathrm{C}$ during $60 \mathrm{~min}$ in a furnace under $\mathrm{N}_{2}$ flow $\left(100 \mathrm{~cm}^{3} \mathrm{~min}^{-1}\right)$. The resulting carbons were ground in a mill, washed with pure water and finally dried at $120^{\circ} \mathrm{C}$. The dried powders were sieved in the size range from 180-250 $\mu \mathrm{m}$.

\section{Kinetics models}

In order to investigate the mechanism of sorption and potential controlling steps such as mass transport, several kinetic models were tested including the pseudo first order kinetic model, the Elovich model and the pseudo second order kinetic model for a batch contact time process, where the rate of sorption of dye on to the given adsorbent is proportional to the amount of dye sorbed from the solution phase.

\section{Pseudo first order kinetic model}

For a batch contact time process, where the rate of sorption of dye on to the given adsorbent is proportional to the amount of dye sorbed from the solution phase, the first order kinetic equation ${ }^{9,10}$ may be expressed as:

$$
\frac{d q_{t}}{d t}=k_{L}\left(q_{e}-q_{t}\right)
$$

Where, $\mathrm{q}_{\mathrm{e}}=$ the amount of dye adsorbed at equilibrium, $\mathrm{mg} / \mathrm{g}, q_{t}=$ the amount of dye adsorbed at time $t, \mathrm{mg} / \mathrm{g}, k_{L}=$ the rate constant for pseudo first order adsorption, $\mathrm{min}^{-1}$. After integration and applying boundary conditions, viz, the initial conditions are $\left(q_{e}-q_{t}\right)=0$ at $t=0$, equation becomes:

$$
\log \left(q_{e}-q_{t}\right)=\log q_{e}-\frac{k_{L}}{2.303} t
$$


The values of $\log \left(q_{e}-q_{t}\right)$ were linearly correlated with t. The plot of $\log \left(q_{e}-q_{t}\right)$ vs. $t$ should give a linear relationship (Lagergren plot) from which $k_{l}$ and $q_{e}$ can be determined from the slope and intercept of the plot, respectively.

\section{The Elovich model}

The Elovich or Roginsky-Zeldovich equation is generally expressed as follows ${ }^{11,12}$.

$$
\frac{d q_{t}}{d t}=\alpha \exp \left(\beta q_{t}\right)
$$

Where, $\mathrm{q}_{\mathrm{t}}=$ the amount of dye adsorbed $\mathrm{mg} / \mathrm{g}$, at time $\mathrm{t}, \alpha=$ the initial dye sorption rate, $\mathrm{mg} / \mathrm{g}, \mathrm{min}, \beta=$ the desorption constant, $\mathrm{g} / \mathrm{mg}$, during any one experiment. To simplify the Elovich equation, Chien ${ }^{13}$ assumed $\alpha \beta \mathrm{t} \gg>1$ and on applying the boundary conditions $\mathrm{q}_{\mathrm{t}}=\mathrm{o}$ at $\mathrm{t}=\mathrm{o}$ and $\mathrm{q}_{\mathrm{t}}=\mathrm{q}_{\mathrm{t}}$ at $\mathrm{t}=\mathrm{t}$, equation becomes

$$
q t=\frac{1}{\beta} \ln (\alpha \beta)+\frac{1}{\beta} \ln t
$$

This constant can be obtained from the slope and intercept of linear plot of $\mathrm{q}_{\mathrm{t}}$ versus $\ln t$. The equation will be used to test the applicability of the Elovich equation to the kinetics of chosen adsorbent adsorbate system.

\section{Pseudo second order kinetic model}

To describe dye adsorption, the modified pseudo second order kinetic equation is expressed as ${ }^{13}$.

$$
\frac{d q_{t}}{d t}=k_{2}\left(q_{e}-q_{t}\right)^{2}
$$

Where, $\mathrm{q}_{\mathrm{e}}=$ the amount of dye adsorbed at equilibrium, $\mathrm{mg} / \mathrm{g}, q_{t}=$ the amount of dye adsorbed at time $t, \mathrm{mg} / \mathrm{g}, K_{2}=$ the rate constant for pseudo first order adsorption, g/mg. min. For the boundary conditions $t=0$ to $t=1$ and $q_{t}=0$ to $q_{t}=q_{t}$, the integrated form of equation obtained is

$$
\frac{1}{\left(q_{e}-q_{t}\right)}=\frac{1}{q_{e}}+k_{2} t
$$

Rearranging the terms in the above equation, we get

$$
q_{t}=\frac{t}{\frac{1}{k_{2} q_{e}{ }^{2}}+\frac{1}{q_{e}}}
$$

This is the integrated rate law for a pseudo second order reaction. This has a linear form is given below:

$$
t / q_{t}=\frac{t}{k_{2} q_{e}{ }^{2}}+\frac{1}{q_{e}} t
$$

In the limit $q_{t} / t \longrightarrow 0$, the initial sorption rate, $\mathrm{h}$, is given by $k_{2} q_{e}{ }^{2}, \mathrm{mg} / \mathrm{g} \min$. Plot of $t / \mathrm{q}_{\mathrm{t}}$ versus $t$ of the above equation should give a linear relation ship with a slop of $1 / q_{e}$ and an intercept of $\frac{1}{k_{2} q_{e}{ }^{2}}$.

A relatively high value of the co-efficient of determination $\left(\mathrm{R}^{2}\right)$ and low standard error of estimate (SE) was used as criteria for the best fit ${ }^{14}$. The regression coefficient $\left(\mathrm{R}^{2}\right)$ and standard error of estimate were calculated as follows: 


$$
\begin{aligned}
R^{2} & =\frac{\sum q^{2}-\sum\left(q-q^{\prime}\right)^{2}}{\sum q^{2}} \\
S E & =\left[\sum\left(q-q^{\prime}\right)^{2} /(N-2)\right]^{0.5}
\end{aligned}
$$

Where, $q$ and $q$ ' are the measured and calculated amounts of dye adsorbed on chosen adsorbent respectively, at time $t$ and $N$ is the number of measurements ${ }^{15}$.

\section{Adsorption thermodynamics}

Any chemical system tends to attain a state of equilibrium from one of non - equilibrium. The thermodynamic parameters, which characterize the equilibrium of the system are the Gibbs free energy change $\Delta \mathrm{G}$, the enthalpy change $\Delta \mathrm{H}$ and the entropy change $\Delta \mathrm{S}$. These parameters were determined using the following relations ${ }^{16}$.

$$
K_{c}=C_{A e} / C_{e}, G=-R T \ln K_{c}, \log K_{c}=\Delta S / 2.303 R-\Delta H / 2.303 R T
$$

Where $K_{c}$ is the equilibrium constant, $C_{A e}$ is the solid phase concentration at equilibrium, $C_{e}$ is the residual concentration at equilibrium, $\mathrm{R}$ is the gas constant $(\mathrm{J} / \mathrm{mole})$ and $T$ is the temperature in Kelvin.

\section{Results and Discussion}

\section{Optimum $\mathrm{pH}$}

The removal of Reactive Red 2 by the activated carbon prepared from Pongamia pinnata seed shell at different $\mathrm{pH}$ at an initial concentration of $20 \mathrm{mg} / \mathrm{L}$, at temperature $30{ }^{\circ} \mathrm{C}$, particle size of 180 - 250 microns are shown in Figure 1.

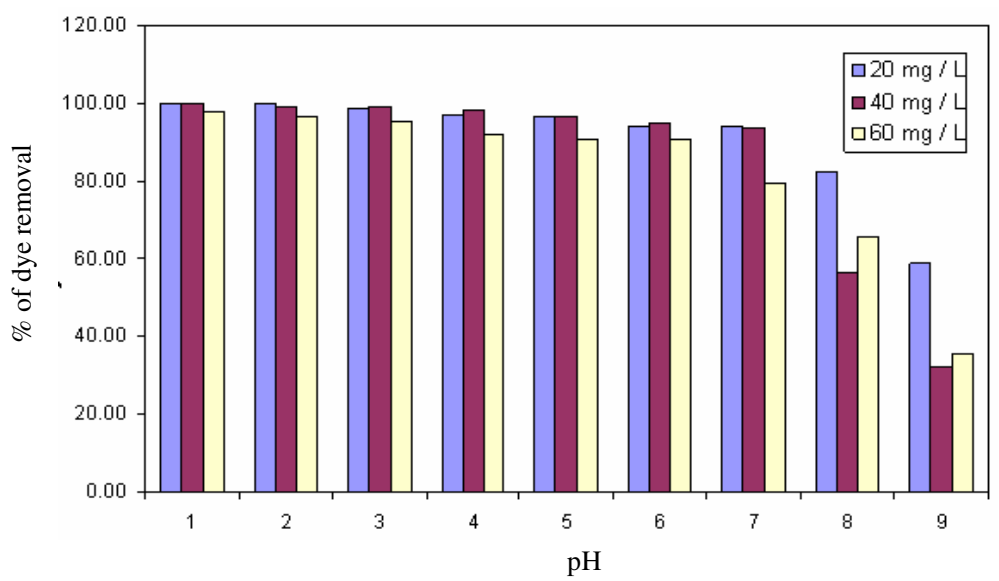

Figure 1. Effect of $\mathrm{pH}$ on \% of dye removal

Results indicate that the chosen adsorbent adsorbs maximally at a $\mathrm{pH}$ of around 6.0 to 7.5 and thereafter, a marginal decrease is observed for the rest of the range. Therefore, the $\mathrm{pH}$ range of 6.5 appears to be the most favorable range for the removal of Reactive Red 2 from aqueous solution with Pongamia pinnata seed shell as an adsorbent.

\section{Adsorption kinetics}

In order to investigate the mechanism of sorption and potential rate-controlling steps such as mass transport and chemical reaction processes, several kinetic models were tested including the pseudo first order, pseudo second order and Elovich equation. 


\section{Effect of initial concentration on kinetics rate constant and rate parameters}

The batch adsorption experiments were carried out by using various concentrations of dyes viz., 20, 40 and $60 \mathrm{mg} / \mathrm{L}$ at $\mathrm{pH} 6.5$ at the reaction temperature of $30{ }^{\circ} \mathrm{C}$. The rate constant and rate parameters at different initial dye concentration presented in the Table 1 showed that the rate constants decreased with better increase in initial dye concentration.

Table 1. Rate parameters for the adsorption of Reactive Red 2 onto Pongamia pinnata seed shell waste activated carbon at various initial concentrations

\begin{tabular}{cccccccccc}
\hline \multirow{2}{*}{$\begin{array}{c}\text { Concentration, } \\
\mathrm{mg} / \mathrm{L}\end{array}$} & \multicolumn{2}{c}{$\begin{array}{c}\text { Pseudo } \\
\text { first order }\end{array}$} & \multicolumn{2}{c}{ Elovich } & \multicolumn{5}{c}{ Pseudo second order } \\
\cline { 2 - 10 } & $\begin{array}{c}\mathrm{K}_{\mathrm{L}}, \\
\mathrm{min}^{-1}\end{array}$ & $\mathrm{R}^{2}$ & $\begin{array}{c}\beta \\
\mathrm{g} \mathrm{min}^{-1}\end{array}$ & $\begin{array}{c}\alpha, \mathrm{mg} \mathrm{g}^{-1} \\
\mathrm{~min}^{-1}\end{array}$ & $\mathrm{R}^{2}$ & $\begin{array}{c}\mathrm{q}_{\mathrm{e}} \\
\mathrm{mg} \mathrm{g}^{-1}\end{array}$ & $\begin{array}{c}\mathrm{K}_{2}, \mathrm{~g} \mathrm{mg}^{-1} \\
\mathrm{~min}^{-1}\end{array}$ & $\begin{array}{c}\mathrm{h}, \mathrm{g} \mathrm{mg}^{-1} \\
\mathrm{~min}^{-1}\end{array}$ & $\mathrm{R}^{2}$ \\
\hline 20 & 0.0099 & 0.98 & 1.0526 & 9.6571 & 0.89 & 8.8888 & 0.01265 & 1 & 0.88 \\
40 & 0.0093 & 0.99 & 0.425 & 6.808 & 0.92 & 21.6666 & 0.00118 & 1.8 & 0.94 \\
60 & 0.0083 & 0.99 & 0.25 & 5.1356 & 0.91 & 34.5454 & 0.00038 & 2.2 & 0.91 \\
\hline
\end{tabular}

An analysis of the data reveals that the influence of the initial concentration of Reactive Red 2 has very little influence on the pseudo first order rate constant. It also reveals that the influence of the initial concentration of the Reactive Red 2 on the Elovich and pseudo second order rate constant is neither appreciable nor very little (Figures 2a, 2b, 2c).

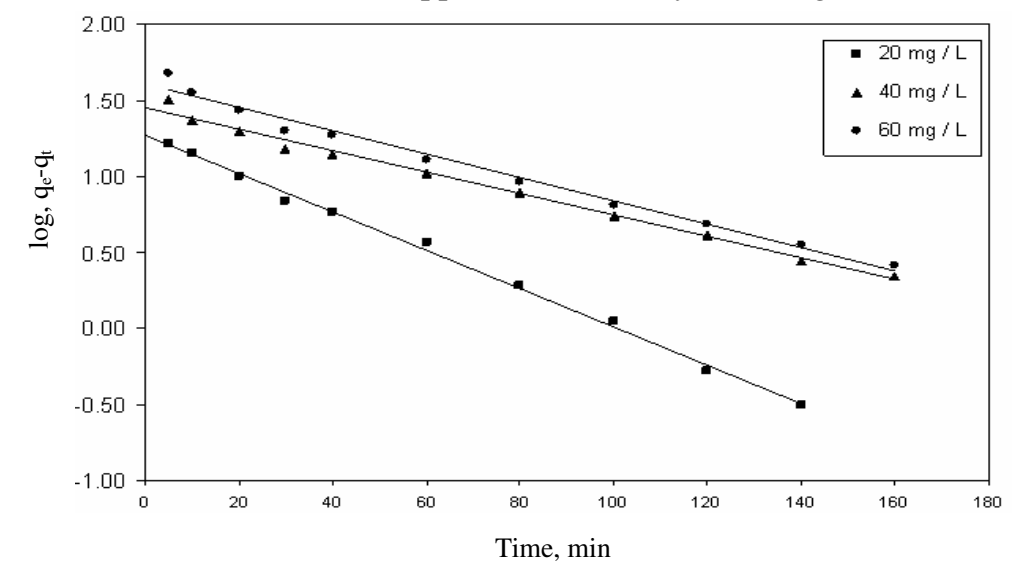

Figure 2a. Pseudo first order plot for Reactive Red 2 removal - initial dye concentration variation

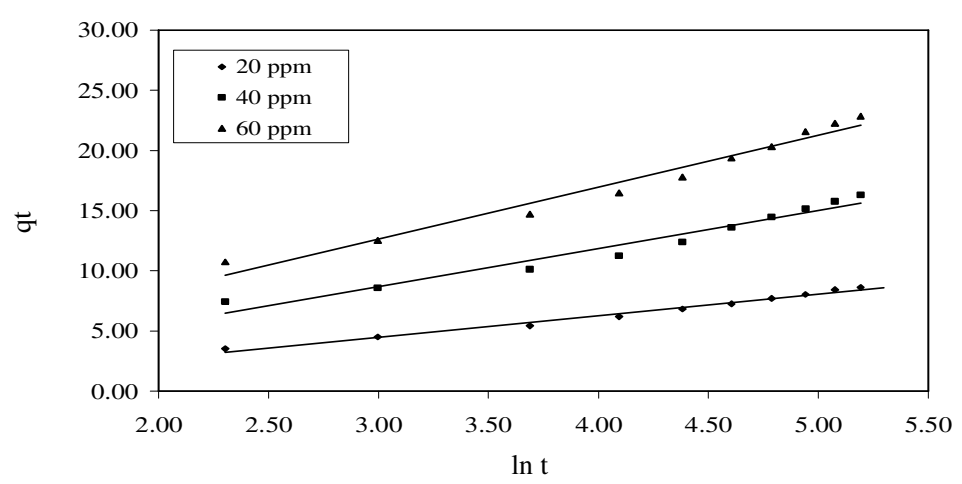

Figure 2b. Elovich plot of Reactive Red 2 removal - initial dye concentration variation 


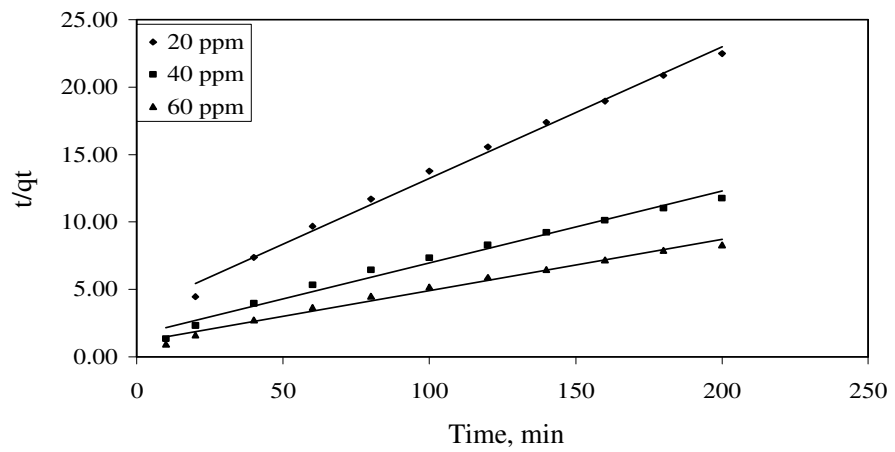

Figure 2c. Pseudo second order plot for Reactive Red 2 removal - initial dye concentration variation

\section{Effect of temperature on kinetic rate constant and rate parameters}

The experiments were conducted at different temperatures viz., 30, 45 and $60{ }^{\circ} \mathrm{C}$. From the slope of each linear trace, the rate constants were calculated and the results are presented in the Table 2. The data obtained separately for each of the kinetic models from the slopes of plots show a good compliance with the pseudo First order equation the $\mathrm{R}^{2}$ values for the linear plots being $>0.985$ showed that kinetic data fitted the pseudo first order adsorption kinetic equation (Figures 3a, 3b, 3c).

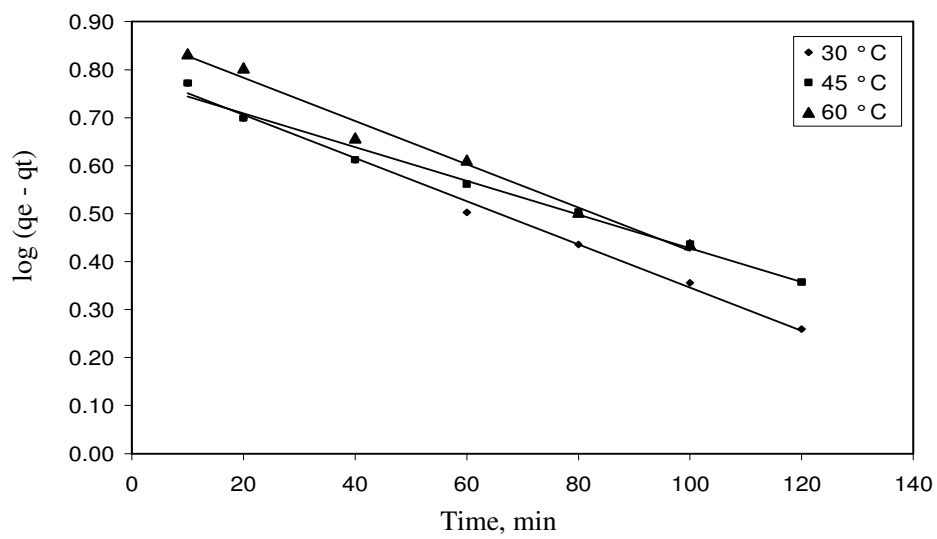

Figure 3a. Pseudo first order plot - temperature variation

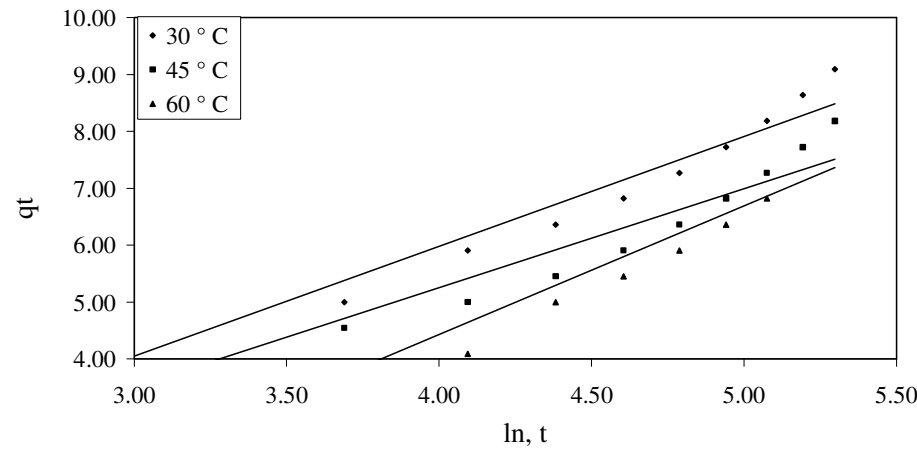

Figure 3b. Elovich plot - temperature variation 


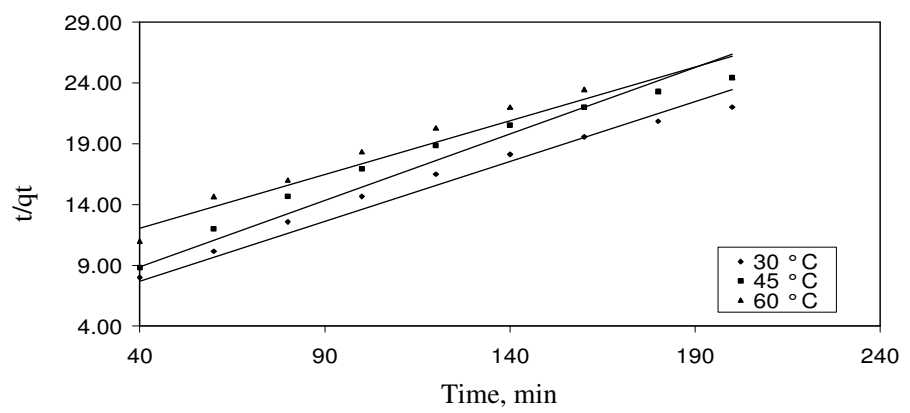

Figure 3c. Pseudo second order plot - temperature variation

Table 2. Rate parameters for the adsorption of Reactive Red 2 onto Pongamia pinnata seed shell waste activated carbon at various temperatures

\begin{tabular}{|c|c|c|c|c|c|c|c|c|c|}
\hline \multirow{2}{*}{ 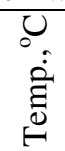 } & \multicolumn{2}{|c|}{$\begin{array}{c}\text { Pseudo first } \\
\text { order }\end{array}$} & \multicolumn{3}{|c|}{ Elovich } & \multicolumn{4}{|c|}{ Pseudo second order } \\
\hline & $\begin{array}{l}\mathrm{K}_{\mathrm{L}}, \\
\min ^{-1}\end{array}$ & $\mathrm{R}^{2}$ & $\begin{array}{c}\beta \\
\mathrm{g} \mathrm{\operatorname {min } ^ { - 1 }}\end{array}$ & $\begin{array}{c}\alpha \mathrm{mg} \mathrm{g}^{-1} \\
\min ^{-1}\end{array}$ & $\mathrm{R}^{2}$ & $\begin{array}{c}\mathrm{q}_{\mathrm{e}} \\
\mathrm{mg} \mathrm{g}^{-1}\end{array}$ & $\begin{array}{c}\mathrm{K}_{2} \mathrm{~g} \mathrm{mg}^{-1} \\
\min ^{-1}\end{array}$ & $\begin{array}{l}\mathrm{h} \mathrm{g} \mathrm{mg} \mathrm{g}^{-1} \\
\mathrm{~min}^{-1}\end{array}$ & $\mathrm{R}^{2}$ \\
\hline 30 & 0.01223 & 0.98 & 0.923 & 3.2795 & 0.93 & 9.221 & 0.00617 & 2 & 0.96 \\
\hline 45 & 0.00904 & 0.99 & 0.8636 & 1.6355 & 0.89 & 10.2857 & 0.0027 & 3.5 & 0.95 \\
\hline 60 & 0.00837 & 0.99 & 0.7692 & 1.5161 & 0.88 & 11.555 & 0.00115 & 6.5 & 0.95 \\
\hline
\end{tabular}

Mechanism for sorption of Reactive Red 2 on to Pongamia pinnata seed shell

Because of the high correlation coefficients obtained using pseudo first order and pseudo second order models, it was impossible to concludes which adsorption mechanism actually occurred and was responsible for the ability of adsorbent to review other sources of information in an attempt to identify the specific adsorption mechanism.

In adsorption process of dye ion on the solid surface, the dye species migrate towards the surface of the adsorbent. This type of migration proceeds till the concentration of the adsorbate species, adsorbed, on to the surface of the adsorbent. Once equilibrium is attained, the migration of the solute species from the solution stops. Under this situation, it is possible to measure the magnitude of the distribution of the solute species between the liquid and solid phases. The magnitude of this kind of distribution is a measure of the efficiency of the chosen adsorbent in the adsorbate species.

When a powdered solid adsorbent material is made in contact with a solution containing dyes, the dyes first migrate from the bulk solution to the surface of the liquid film. This surface exerts a diffusion barrier. This barrier may be very significant or less significant ${ }^{17}$. The involvement of a significant quantum of diffusion barrier indicates the dominant role taken up by the film diffusion in the adsorption process. Furthermore, the rate of an adsorption process is controlled either by external diffusion, internal diffusion or by both types of diffusions.

The external diffusion controls the migration of the solute species from the solution to the boundary layer of the liquid phase. However, the internal diffusion controls the transfer of the solute species from the external surface of the adsorbent to the internal surface of the pores of the adsorbent material ${ }^{18}$. It is now well established, that during the adsorption of dye over a porous adsorbent, the following three consecutive steps were taken place ${ }^{19}$.

(i) Transport of the ingoing adsorbate ions to external surface of the adsorbent (film diffusion),

(ii) Transport of the adsorbate ions within the pores of the adsorbent except for a small amount of adsorption, which occurs on the external surface (particle diffusion) and

(iii) Adsorption of the ingoing adsorbate ions on the interior surface of the adsorbent ${ }^{20}$. 
Out of these three processes, the third process is considered to be very fast and is not the rate limiting step in the uptake of organic compounds ${ }^{21}$. The remaining two steps impart the following three possibilities:

Case 1: External transport > internal transport, where rate is governed by particle diffusion.

Case 2: External transport < internal transport, where the rate is governed by film diffusion.

Case 3: External transport $\approx$ internal transport, which accounts for the transport of the adsorbate ions to the boundary and may not be possible within a significant rate, which later on gives rise to the formation of a liquid film surrounded by the adsorbent particles with a proper concentration gradient.

In the batch-mode contact time adsorption experiments, rapid stirring was maintained. This Reactive Red 2 to the transport of the adsorbed species from the solution to the pores of the adsorbent material and this step may control the rate of the adsorption process.

According to Michelson et al., ${ }^{21}$ for film diffusion to be rate-determining step, the value of the film diffusion coefficient, $D_{\mathrm{f}}$, should be in the range $10^{-6}-10^{-8} \mathrm{~cm}^{2} / \mathrm{sec}^{22}$. If pore diffusion were to the rate limiting, the pore diffusion coefficient, $D_{p}$, should be in the range $10^{-11}-10^{-13} \mathrm{~cm}^{2} / \mathrm{sec}$. In order to find out the nature of the process responsible for adsorption on to chosen adsorbent, attempts were made to calculate the diffusion co-efficient of the process. Assuming spherical geometry of the sorbents ${ }^{23}$, the overall rate constant of the process can be correlated to the pore diffusion coefficient in accordance with the expression,

$$
t_{1 / 2}=0.03 \frac{r_{o}^{2}}{D_{p}} \text { or to the film diffusion coefficient in accordance with } t_{1 / 2}=0.23 \frac{r_{o} \partial}{D_{f}} \times \frac{\bar{C}}{C}
$$

Where $r_{o}$ is radius of the sorbent $(\mathrm{cm}), D_{p}$ and $D_{f}$ are pore diffusion coefficient $\left(\mathrm{cm}^{2} / \mathrm{sec}\right)$ and film diffusion coefficient $\left(\mathrm{cm}^{2} / \mathrm{sec}\right)$ respectively, $\frac{\bar{C}}{C}$ is equilibrium loading of the adsorbent, $\partial$ is the film thickness $(\mathrm{cm})$ and $\mathrm{t}_{1 / 2}$ is the time for half change $(\mathrm{sec})$.

Since the carbon particles used were of the size range $(180-250 \mu \mathrm{m})$, the average diameter of the particle was taken as $0.0215 \times 10^{-4} \mathrm{~cm}$. Using these values, the film diffusion coefficients and pore diffusion coefficients were calculated.

Then considering the pseudo first order rate constant $\mathrm{k}_{\mathrm{L}}$, for the adsorption of Reactive Red 2. The values of $D_{p}$ and $D_{f}$ were calculated under the given set of operating conditions, and are presented in the Table 3.

Table 3. $D_{p}$ and $D_{f}$ values for the chosen adsorbent - adsorbate system

\begin{tabular}{ccccc}
\hline Dye & Temperature, ${ }^{\circ} \mathrm{C}$ & $\mathrm{t}_{1 / 2}, \mathrm{~s}$ & $\mathrm{D}_{\mathrm{p}} \times 10^{-10}, \mathrm{~cm}^{2}$ & $\mathrm{D}_{\mathrm{f}}, \times 10^{-9}, \mathrm{~cm}^{2}$ \\
\hline \multirow{2}{*}{ Reactive } & $30{ }^{\circ} \mathrm{C}$ & 3398.72 & 1.0200 & 6.613 \\
Red 2 & $45{ }^{\circ} \mathrm{C}$ & 4595.99 & 0.7543 & 4.645 \\
& $60{ }^{\circ} \mathrm{C}$ & 4695.37 & 0.6931 & 4.073 \\
\hline
\end{tabular}

The values of $D_{p}$ and $D_{f}$ for all Reactive Red 2 in the present study are found to be in the order of $10^{-10}$ to $10^{-11}$ and $10^{-9}$ to $10^{-10} \mathrm{~cm} / \mathrm{sec}$ which indicates that pore diffusion has some amount of influence in the rate determining step.

Gupta et al, ${ }^{24}$ have reported a $D_{p}$ value in the order of $10^{-10} \mathrm{~cm} / \mathrm{sec}$ for the removal of Chrome Red 2 by a mixture of two adsorbents and these authors suggested that the adsorption process was governed by pore diffusion even though it could not be ascertained that pore diffusion was the only rate determining step. 
The present study indicates the $D_{p}$ values in the order of $10^{-11}$ to $10^{-10} \mathrm{~cm} / \mathrm{sec}$ and the $D_{f}$ values in the order of $10^{-10}$ to $10^{-9}$ for the respective Lagergren plots for Reactive Red 2 and hence, the investigator concludes that the mechanism of the removal of Reactive Red 2 in the present study by chosen adsorbent is complex.

Since both external Mass transfer and intra-particle diffusion constants varied with initial Reactive Red 2 concentration indicating the occurrence of both surface adsorption and intra particle diffusion, the sorption data were further analyzed by the kinetic expression given by Boyd et $a l^{25}$.

$$
F=1-\frac{6}{\pi^{2}} \sum_{N-1}^{\alpha} \frac{1}{n^{2}} \exp \left[\frac{-D i t \pi^{2} n^{2}}{r^{2}}\right] \quad F=1-\frac{6}{\pi^{2}} \sum_{N-1}^{\alpha} \frac{1}{n^{2}} \exp \left[-n^{2} \beta_{t}\right]
$$

Where $F$ is the fractional attainment of equilibrium at time $t$ and is obtained by using following equation and $n$. is the Freundlich constant of the adsorbate.

$$
F=\frac{q_{t}}{q_{e}}
$$

Where $q_{t}$ and $q_{e}$ are the amounts adsorbed at time $t$ and at equilibrium respectively. On the basis of $F$ values, corresponding values of $B_{t}$ were obtained from Reichenberg's table ${ }^{26}$ and the linearity test was carried out by plotting $B_{t}$ with respect to time for both the solutions at different time intervals and at 30,45 and $60{ }^{\circ} \mathrm{C}$. The linearity test of $B_{t}$ versus time plot drawn for different concentrations is employed to distinguish between film diffusion and particle diffusion. From the slop of the straight line obtained from time versus $B_{t}$ graph, the $B$ value (time constant) were calculated. The values of effective diffusion coefficient $\left(D_{i}\right)$ were calculated at different temperatures using the following Equation. $B=\pi^{2} \mathrm{D}_{\mathrm{i}} / \mathrm{r}^{2}$, here ' $\mathrm{r}$ ' is the radius of the absorbent particle. The $D i$ values are given in the Table 4.

Table 4. Values of energy of activation $E_{a}$, entropy of activation $\Delta S^{\#}$ and pre-exponential constant $D_{o}$ for the present study

\begin{tabular}{ccc}
\hline S. No. & Parameter & Value \\
\hline 1. & $D_{i}, \mathrm{~cm}^{2} \mathrm{~s}^{-1}$ & $1.3876 \times 10^{-11}$ \\
& $30^{\circ} \mathrm{C}$ & $1.2728 \times 10^{-11}$ \\
& $45^{\circ} \mathrm{C}$ & $1.0341 \times 10^{-11}$ \\
2. & $60^{\circ} \mathrm{C}$ & -9.5735 \\
3. & $E_{a}, \mathrm{~kJ} \mathrm{~mol}^{-1}$ & -185.02 \\
4. & $\Delta S^{\#}, \mathrm{JK}^{-1} \mathrm{~mol}^{-1}$ & $9.3325 \times 10^{-12}$ \\
\hline
\end{tabular}

The plot of $1 / T$ versus $\log D_{i}$ was found linear with negative slope indicating thereby the increase in the mobility of ions. This is due to the fact that with the rise in temperature the mobility of ions. This is due to the fact that with the rise in temperature the mobility of ions increases, which consequently decreases the retarding force acting on the diffusing ions. The Values of energy of activation $E_{a}$, entropy of activation $\Delta \mathrm{S}^{\#}$ and pre-exponential constant $D_{o}$ were calculated using following Equations.

$$
D_{i}=D_{o} \exp \left[-E_{a} / R T\right], D_{o}=\left(2.72 d^{2} k T / h\right) . \exp \left[\Delta S^{\#} / R\right]
$$

Where $\mathrm{d}$ is the average distance between the successive exchange sites and is taken as $5 \mathrm{~A}^{\circ} . \mathrm{R}, \mathrm{h}$ and $\mathrm{k}$ are the Gas, Planck and Boltzmann constants, respectively. The values of $E_{a}, D_{i}, D_{o}, \Delta S^{\#}$ and other parameters are given in Table 4 . The negative values of $\Delta S^{\#}$ reflects that no significant change occurs in the internal structure of chosen adsorbent during the adsorption process. 


\section{Conclusion}

In the present study adsorption of Reactive Red 2 on activated Pongamia pinnata seed shell waste carbon has been investigated. The data obtained through this work supports that the Pongamia pinnata waste carbon is an effective low cost adsorbent for the removal Reactive Red 2 from aqueous solution. The adsorption of Reactive red 2 is dependent on the initial concentration and agitation time. Equilibrium of reactive red 2 adsorption reaches at $160 \mathrm{~min}$.

The pseudo first and second order equations provide a best fit description for the sorption of Reactive Red 2 on to Pongamia pinnata related to Elovich model, but the pseudo first order correlation co-efficient has better correlation value than pseudo second order equation, pseudo first order equation is consider to be the most appropriate due to high correlation coefficient when compared to pseudo second order equation. The adsorption of Reactive Red 2 on to activated Pongamia pinnata waste carbon is an exothermic reaction based on enthalpy change values.

\section{References}

1. Anjusingh and Ramishukla, Poll Res., 2000, 19, 179.

2. Nagarnaik P B, Bhole A G and Natarajan G S, Indian J Environ Health, 2003, 45, 1-4.

3. Sharma Y C, Uma, Srivastava V, Srivastava J and Mahto M, Chem Eng J., 2007, 127, $151-156$

4. Namasivayam C and Kadirvelu K, Environ Technol., 2000, 21, 1091-1097.

5. Karthikeyan S, Sivakumar P and Palanisamy P N, E-J Chem., 2008, 5, 409.

6. Kaviyarasan V, Mohan N, Kannan V,Ebenezer P and Karthikeyan S, Poll Res.,2003, 22, 77-80.

7. Karthikeyan S, Jambulingam M, Sivakumar P, Shekhar A P and Krithika J, E-J Chem., 2006, 3(4), 303-306.

8. Ho Y S and McKay G, Can J Chem Engg., 1998, 76(4), 822-827.

9. Karthikeyan S, Bhuwaneshwari G, Malathi S and Maheswari P, J Indian Coun Chemists, 2007, 24, 63.

10. Low K S, Lee C K and Ng A Y, Biores Technol., 1999, 68, 205-208.

11. Karthikeyan S, Jambulingam M and Sivakumar P, Res J Chem Environ., 2006, 10(4), 72-80.

12. Ho Y S, McKay G and Wasedaj Foster C F, Adsorpt Sci Technol., 2000, 18, 639-650.

13. Chein S H and Clayton W R, Soil Sci Soc Am J., 1980, 44, 265-268.

14. Steel R D G and Torri J H, 1969 Principles and Procedures of Statistics McGraw Hill Co Newyork, 1960, 481.

15. Stephen Inbaraj and Sulochana N, Indian J Chem Technol., 2002, 9, 201-208.

16. Weber W J Jr and Miller C T, Water Res., 1998, 22(4), 457-464.

17. Gupta V K, Mittal A and Gajbe V, J Colloid Interface Sci., 2005, 284, 89-98.

18. Gupta V K, Ali I, Suhas and Mohan D, J Colloid Interface Sci., 2003, 265, 257-264.

19. Crank J ( $\left.2^{\text {nd }} E d\right)$, The Mathematics of Diffusion, Clarendon, Oxford, 1975.

20. Weber W J Jr and Morris C J, J Sanit Eng Div., 1963, 89, 31-60.

21. Michelson L D, Gideon P G, Pace E G and Kutal L H, Water Res Tech Bull., 1975, 74.

22. Bhattacharya A K and Venkobacher C, J Environ Eng Div., 1984, 110, 110.

23. Gupta G S, Prasad G, Panday K K and Singh V N, Wat Air Soil Pollut., 1988, 37, 13-24.

24. Boyd G E, Adamson A W and Myers L S, J Am Chem Soc., 1947, 69(11), 2836.

25. Reichenberg D, Properties of Ion - Exchange Resins in Relation to their Structure III. Kinetics of Exchange, 1953. 


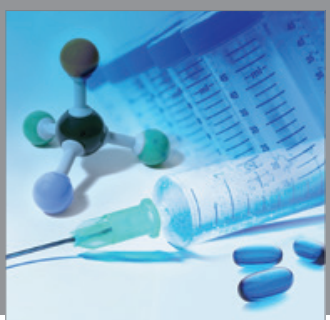

International Journal of

Medicinal Chemistry

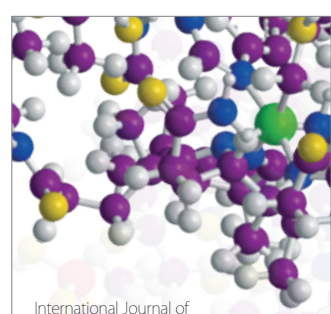

Carbohydrate Chemistry

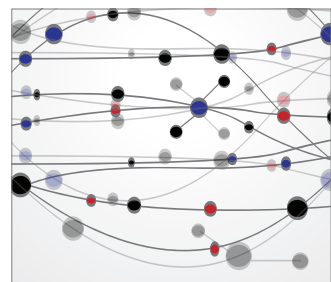

The Scientific World Journal
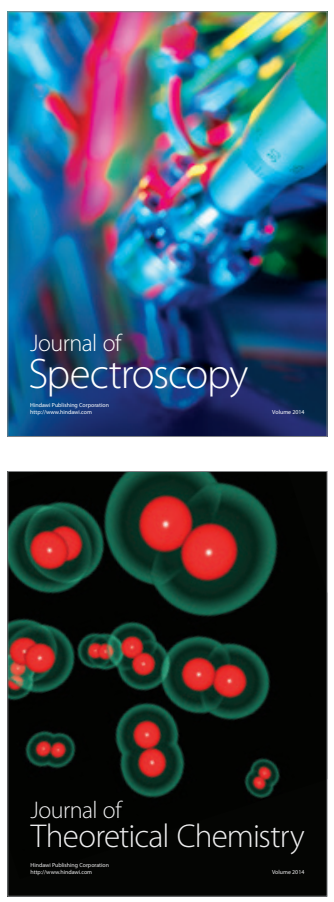
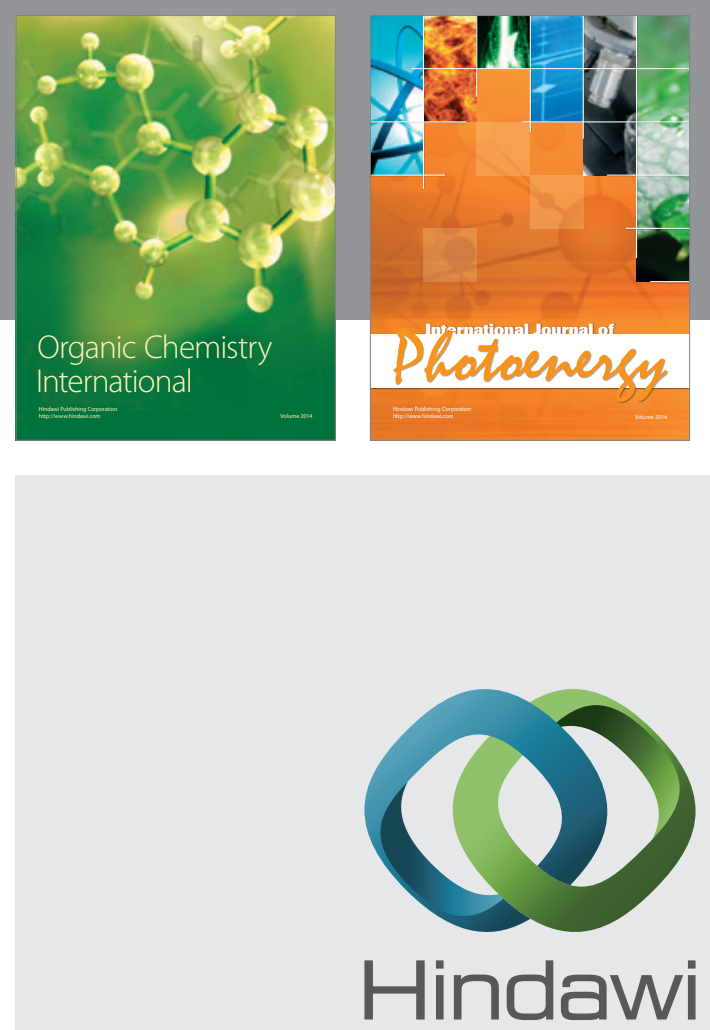

Submit your manuscripts at

http://www.hindawi.com
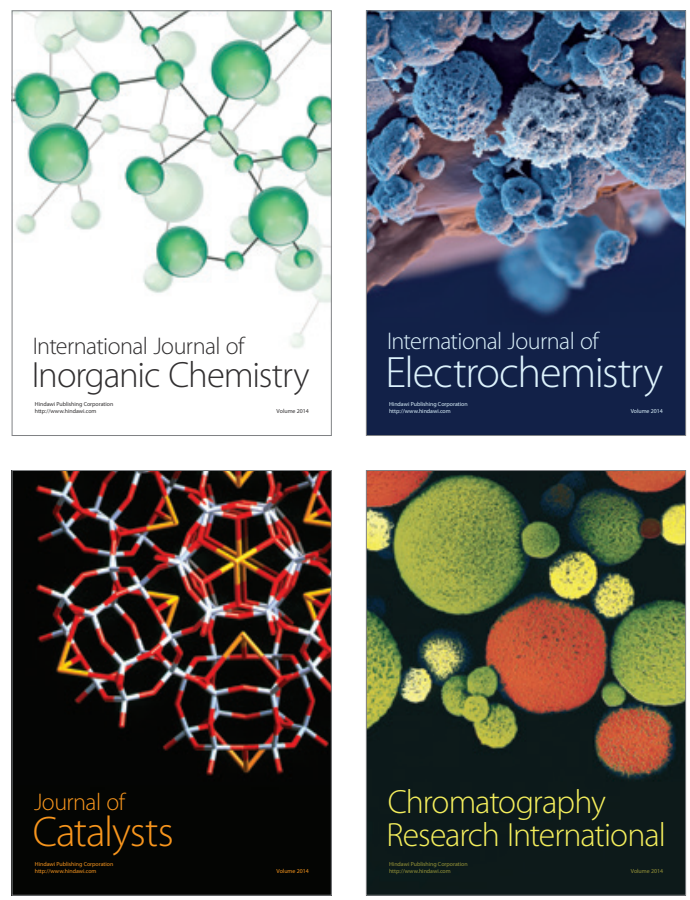
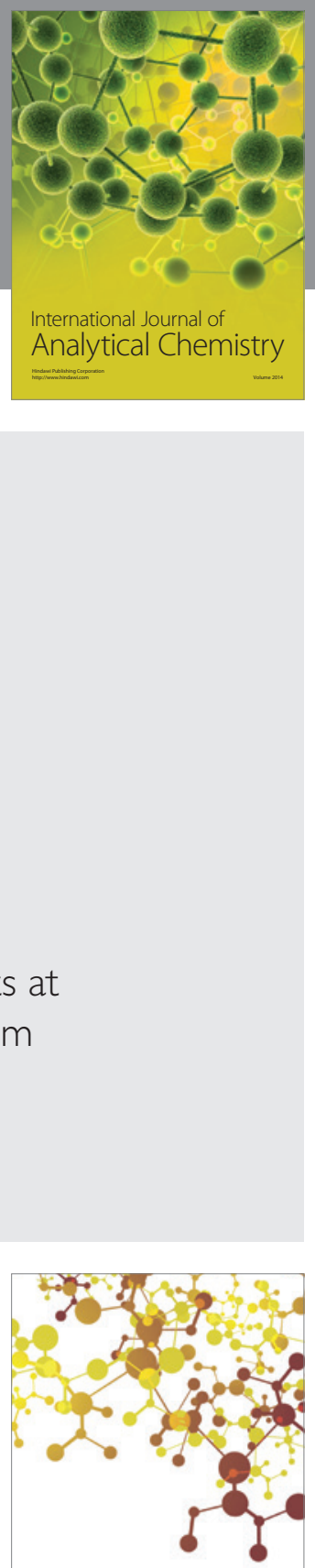

Journal of

Applied Chemistry
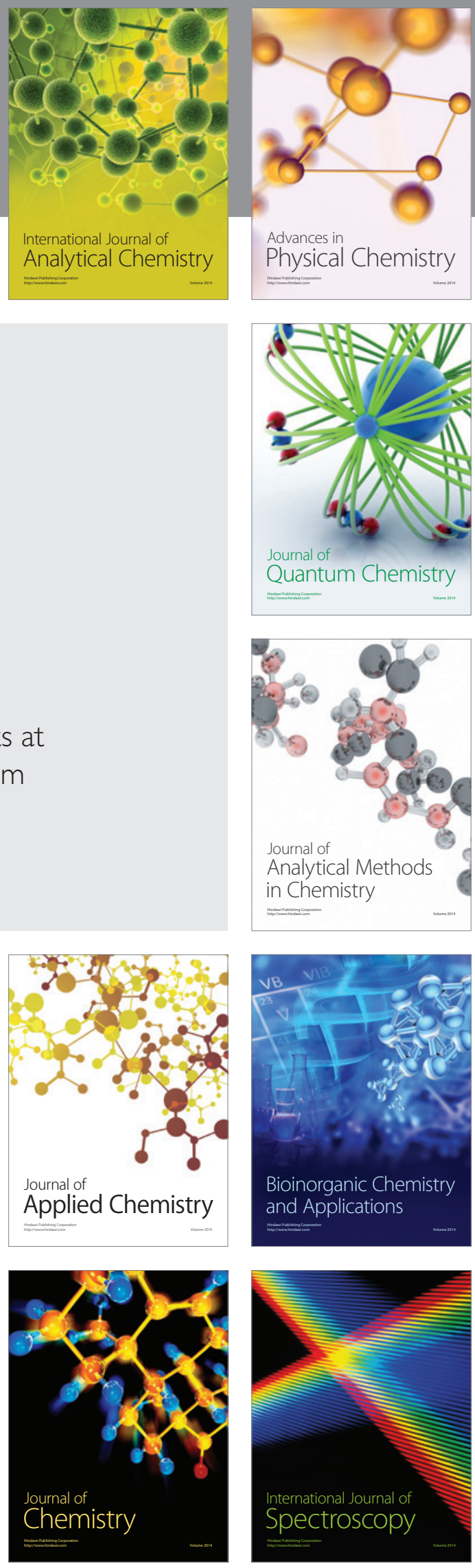